Document downloaded from:

http://hdl.handle.net/10251/170078

This paper must be cited as:

Babiloni, E.; Guijarro, E. (2020). Fill rate: from its definition to its calculation for the continuous $(\mathrm{s}, \mathrm{Q})$ inventory system with discrete demands and lost sales. Central European Journal of Operations Research. 28(1):35-43. https://doi.org/10.1007/s10100-018-0546-7

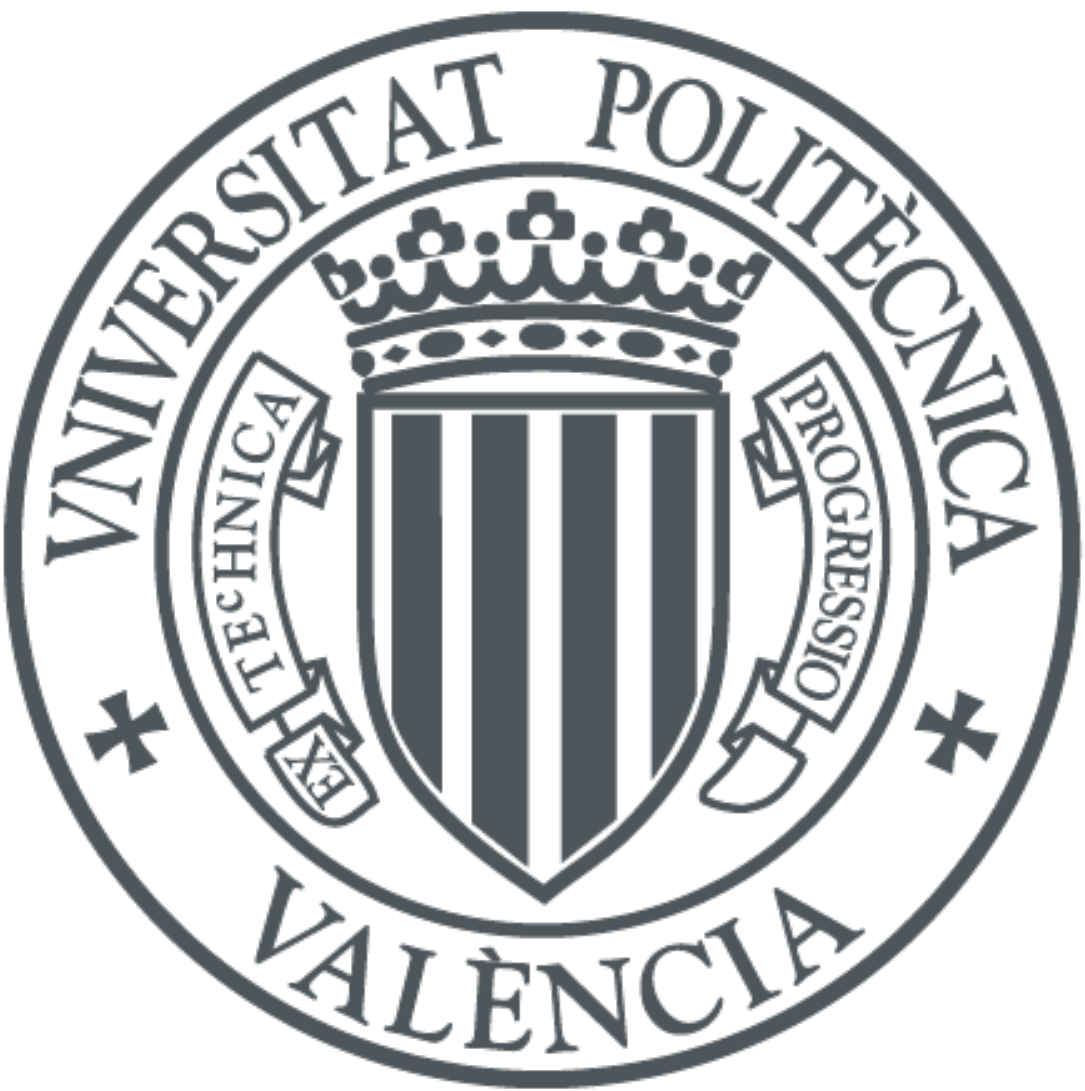

The final publication is available at

https://doi.org/10.1007/s10100-018-0546-7

Copyright Springer-Verlag

Additional Information 


\title{
Fill Rate: from its definition to its calculation for the continuous $(s, Q)$ inventory system with discrete demands and lost sales
}

\author{
Eugenia Babiloni, Ester Guijarro
}

\begin{abstract}
Customer service measures are traditionally used to determine the performance or/and the control parameters of any inventory system. Among them, the fill rate is one of the most widely used in practice and is defined as the fraction of demand that is immediately met from shelf i.e. from the available onhand stock. However, this definition itself set out several problems that lead to consider two different approaches to compute the fill rate: the traditional, which computes the fill rate in terms of units short; and the standard, which directly computes the expected satisfied demand. This paper suggest two expressions, the traditional and the standard, to compute the fill rate in the continuous reorder point, order quantity $(s, Q)$ policy following these approaches. Experimental results shows that the traditional approach is biased since underestimate the real fill rate whereas the standard computes it accurately and therefore both approaches cannot be treated as equivalent. This paper focuses on the lost sales context and discrete distributed demands.
\end{abstract}

Keywords: inventory; continuous review; fill rate; lost sales

\section{Introduction and Literature Review}

In many industries, inventory control has become a strategic key element that determines the success of important objectives. There are several decisions that inventory managers need to adopt for designing inventory policies. First of all it is important to determine how to proceed when the system is temporarily out of stock. If any demand, when out of stock, is backordered and filled as soon as possible, the inventory is managed in the backordering case. Conversely, if once a stockout takes place, the customer goes elsewhere to satisfy its need, the inventory is managed in the lost sales case (Silver, 1998). Inventory research has traditionally focused on the backordering case mainly because it is mathematically much easier to characterize the inventory policy when demand is backorder rather than lost (Bi- 
jvank et al. 2011; Zipkin 2008a; Zipkin 2008b). However, the assumption of excess demand being lost is of practical importance in sectors where customers are impatient. Examples are found in highly competitive sectors such as retail (Gruen et al. 2002), machinery spare parts, service sector (Diels and Wiebach 2011) or on-line commerce (Breugelmans et al. 2006). Thus inventory managers are increasingly demanding methods and models for the lost sales case which is precisely the context of this paper. Once this question has been solved, the fundamental purpose of an inventory system is to resolve the following issues (Silver, 1998): (1) how often the inventory status is reviewed; (2) when a replenishment order takes place and (3) how large the replenishment order is. This paper is focus on one of the most frequently used inventory control system: the traditional continuous review system $(s, Q)$. Contrary to what happens in the periodic review system, where the status of the stock is only known when it is reviewed, in the case of continuous review, it is known at any moment. This fact allows controlling when the inventory position (i.e. on-hand stock + on-order stock - backorders) drops to the reorder point, $s$, at which point a replenishment order equal to $Q$ is launched and received after the lead time. To determine the order point, $s$, and the order quantity, $Q$, we find two possible approaches: minimizing total inventory costs or guaranteeing the achievement of a target service level. As Bijvank and Vis (2012) points out the service approach is useful when a service level restriction is imposed by the replenishment process and it is easier to define a target service level than all the costs, especially the stock out cost. For this reason, practitioners tend to use the service level criterion to establish the control parameters of the inventory system.

One of the service measures most commonly used in practice is the fill rate ( $\beta$ futher on) that represents the fraction of demand that is immediately fulfilled from shelf, i.e. from the on-hand stock (see Axsäter (2000) among others). This simple-looking definition entails many technical details that are sometime overlooked. In the literature, the fill rate has been simplified through the traditional approximation which computes the fill rate in terms of units short, i.e. as the complement of the quotient between the expected unfulfilled demand per replenishment cycle (also known as expected shortage) and the total expected demand. Over the last fifty years and for the continuous review system $(s, Q)$ several authors have suggested methods to compute this measure based on the traditional approach. (Silver 1970) proposes an exact formula for Normal distributed demands which eliminate the double counting of the backorders. (Vincent 1983) reviews the expression proposed by Silver (1970) and suggest practical approaches to the calculation of the fill rate that are easy implementable for both Normal and Poisson demands under the assumptions of unitary demand and backlog. Platt et al. (1997) derive two closed-form heuristic approaches to determine the parameters of this policy in a context of Normal 
demand and backorder. Agrawal and Seshadri (2000) develop an upper and lower bound to the fill rate constrained inventory problem for continuous demands and backorder assumption. Axsäter (2006) propose a simple two-step approach to determine the parameters of the policy when the demand is Normal and excess demand is backordered. However, the results of this paper show that the traditional approach does not really equivalent to the fill rate definition.

To the best of our knowledge, there is not any method to estimate the fill rate following its definition for the lost sales case and when the inventory is continuously reviewed by a $(s, Q)$ policy. This gap motivates the objectives of this research: (i) to analyze in depth the definition of the fill rate; (ii) to demonstrate that the traditional method is not accurate enough; and (iii) to derive a standard approach for the fill rate under the assumption of discrete demand.

The remainder of the paper is organized as follows. Section 2 describes the inventory system and introduces the notation and general assumptions of this paper. Section 3 dedicates to analyze in depth the fill rate definition. Section 4 proposes the traditional and the standard method to compute the fill rate in this research context. Simulation and experimental results are illustrated and discussed in Section 5 that also explains the practical implications that arise from using the two approaches presented in the paper. Finally, Section 6 summarizes the main contributions of this research.

\section{Inventory System, Assumption and Notation}

This paper considers a single echelon single item inventory system where demand is stochastic and modelled by any discrete distribution. The stock is controlled according to a continuous review reorder point, order quantity $(s, Q)$ system for the lost sales case as shown in Fig. 1. In this system, a fixed quantity $Q$ is ordered whenever the inventory position (i.e. on-hand stock + on-order stock backorders) drops to the reorder point $s$. The replenishment order is received $L$ periods after being launched. The replenishment cycle is the time elapsed between two consecutives order deliveries.

General assumptions are: (i) time is discrete and is organized in a numerable and infinite succession of equally spaced instants; (ii) the lead time, $L$, is constant and known; (iii) there is never more than one order outstanding which implies that $\mathrm{s}<\mathrm{Q}$; (iv) a replenishment order of size $Q$ is placed when de inventory position is exactly at the reorder point; (v) the replenishment order is added to the inventory at the end of the period in which it is received, hence these products are available to satisfy the demand of the following period; (vi) demand during a period is fulfilled with the on-hand stock at the begin- 
ning of that period; (vii) excess demand is lost and (viii) the demand process is assumed to be discrete, stationary and i.i.d.

The notations in Fig. 1 and in the rest of the paper are:

$s=$ reorder point, ROP (units),

$Q=$ replenishment order quantity (units),

$L=$ lead time for the replenishment order (time),

$z_{0}=$ on-hand stock at the beginning of the cycle or at order delivery (units),

$D_{L}=$ accumulated demand during the lead time (units),

$D_{\tau}=$ accumulated demand from the beginning of the cycle until the ROP is reached (units),

$f_{t}(\cdot)=$ probability mass function of demand at $t$,

$F_{t}(\cdot)=$ cumulative distribution function of demand during $t$ periods,

$X^{+}=\operatorname{maximum}\{X, 0\}$ for any expression $X$.

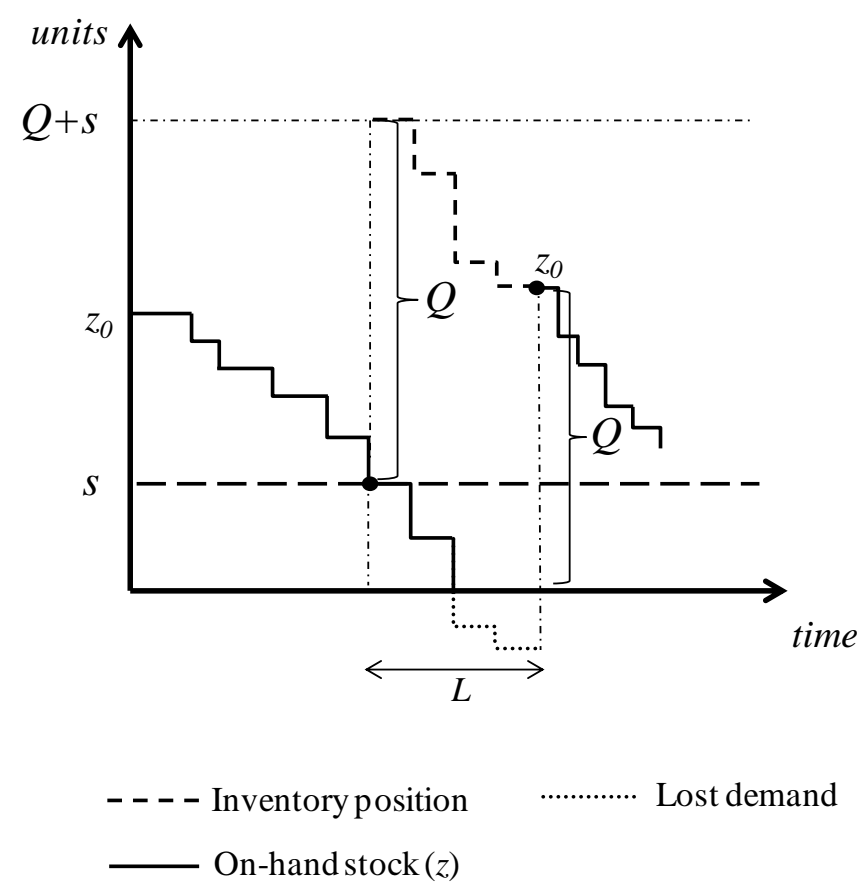

Fig. 1 Evolution of stock in a $(s, Q)$ inventory policy and lost sales. 


\section{3-Fill Rate definition: traditional and standard approach}

The fill rate is one of the service measures most used in practice since it considers not only the possibility that the system is out of stock, but also the size of the unfulfilled demand when it occurs Tempelmeier (2007). As the Introduction section points out, this metric is commonly defined as the fraction of demand that is immediately satisfied from shelf and can be expressed as:

$$
F R=E\left(\frac{\text { fulfilled demand }}{\text { total demand }}\right)
$$

Or by using its complementary:

$$
F R=1-E\left(\frac{\text { unfulfilled demand }}{\text { total demand }}\right)
$$

Although its definition is simple, we find different interpretations of it in the literature and consequently different expression to compute the fill rate. In fact, only few works suggest computing the fill rate following expression (1), i.e. estimating directly the expected fulfilled demand per replenishment cycle. However, it is normally accepted the known as traditional approximation which computes the numbers of units not satisfied, instead of computing directly the fulfilled demand, as:

$$
F R_{\text {Trad }}=1-\frac{E(\text { unfulfilled demand })}{E(\text { total demand })}
$$

However as (Guijarro et al. 2012) point out expressions (2) and (3) are not mathematically equivalent. As known, $E(X \cdot Y)=E(X) \cdot E(Y)$ if $X$ and $Y$ are independent random variables. Even in this context, $E\left(\frac{X}{Y}\right)=E(X) \cdot E\left(\frac{1}{Y}\right)$ but $E\left(\frac{1}{Y}\right) \neq \frac{1}{E(Y)}$ and therefore $E\left(\frac{X}{Y}\right) \neq \frac{E(X)}{E(Y)}$ (see for example Grinstead and Snell (1997)). Therefore, $E\left(\frac{\text { fulfilled demad }}{\text { total demand }}\right) \neq \frac{E(\text { fulfilled demad })}{E(\text { total demand })}$ note that

$$
\begin{aligned}
& \frac{E(\text { fulfilled demand })}{E(\text { total demand })}=1-\frac{E(\text { unfulfilled demand })}{E(\text { total demand })} \text { and then } \\
& E\left(\frac{\text { fulfilled demand }}{\text { total demand }}\right) \neq 1-\frac{E(\text { unfulfilled demand })}{E(\text { total demand })}
\end{aligned}
$$




\section{Fill Rate Estimation in Lost Sales Context for the $(s, Q)$ policy}

This section dedicates to derivate expressions for computing the fill rate when considering the two approaches explained in the previous section: the traditional approximation of the fill rate according to expression (3); and the standard method according to expression (2).

\subsection{Derivation of the Traditional approach}

This approach consists of computing, on one hand, the expected unfulfilled demand and, on the other hand, the expected total demand per replenishment cycle. According to the assumptions of this research, the system will only be out of stock during the lead time and, therefore:

$$
E(\text { Unfilled demand })=\sum_{i=s+1}^{\infty}(i-s) \cdot f_{L}(i)
$$

The expected total demand per replenishment cycle results from adding up the demand from the beginning of the cycle until reaching the ROP and the demand during the lead time. To compute it we need to know the on-hand stock balance at order delivery, which, in a lost sales context, is obtained thus $z_{0}=Q+E\left[s-D_{L}\right]^{+}$. Therefore, the demand that is required to reach exactly the ROP is: $D_{\tau}=z_{0}-s=Q+E\left[s-D_{L}\right]^{+}-s$. Hence,

$$
E(\text { Total demand })=Q+\sum_{j=0}^{s}(s-j) \cdot f_{L}(j)-s+\sum_{k=0}^{\infty} k \cdot f_{L}(k)
$$

Consequently, the traditional approach to calculate the fill rate in a lost sales context that is applicable to any discrete demand distribution is set out as follows:

$$
\beta_{\text {Trad }}=1-\frac{\sum_{i=s+1}^{\infty}(i-s) \cdot f_{L}(i)}{Q+\sum_{j=0}^{s}(s-j) \cdot f_{L}(j)-s+\sum_{k=0}^{\infty} k \cdot f_{L}(k)}
$$

\subsection{Derivation of the Standard approach}

This approach consists of directly computing the fraction of demand that is unfulfilled over the total demand during the replenishment cycle as in expression (2) and following the fill rate definition. Obviously the demand that is lost during the lead time depends on the difference between $D_{L}$ and the on- 
hand stock when the replenishment order is launch, which is exactly equal to $s$. Furthermore, when a stockout takes place the on-hand stock at order delivery in a lost sales context is $z_{0}=Q$ since $E\left[s-D_{L}\right]^{+}=0$. Therefore, the demand that is required to reach exactly the ROP is: $D_{\tau}=z_{0}-s=Q-s$. Then the total demand over the replenishment cycle is equal to $Q-s+D_{L}$.

Therefore the standard method to calculate the fill rate in a lost sales context that is applicable to any discrete demand distribution is as follows:

$$
\beta_{\text {Standard }}=1-\sum_{i=s+1}^{\infty}\left[\left(\frac{i-s}{Q-s+i+F_{L}(s)}\right) \cdot f_{L}(i)\right]
$$

\section{Illustrative Examples and practical implications}

This section illustrates the performance of $\beta_{\text {Trad }}$ and $\beta_{\text {Standard }}$ against a simulated fill rate. Using a randomly generated demand following a Pure Poisson distribution function, we simulate the traditional continuous $(s, Q)$ policy and compute the simulated fill rate, $\beta_{S i m}$ as the average fraction of the complement of the unfulfilled demand in every replenishment cycle when considering 20,000 consecutive periods. This simulation uses the data from Table 1, which encompasses 270 different cases. To assure the consistency of the results, we accomplish thirty replications to each case using the average of these replications as the final $\beta_{\text {Sim }}$.

Table 1 Set of data

Lead Time $L=2,3,4$

Order Quantity $Q=5 ; 6 ; 7 ; 8 ; 9$

Reorder point $s=2 ; 3 ; 4$

Demand Variability (Poisson distributed) $\lambda=0.1 ; 0.5 ; 1 ; 1.5 ; 2 ; 2.5$

Fig. 2 (a) shows the comparison between $\beta_{\text {Trad }}$ and $\beta_{\text {Sim. }}$. As it can be observed, $\beta_{\text {Trad }}$ systematically underestimate $\beta_{\text {Sim. }}$. Fig. 2 (b) shows the comparison between $\beta_{\text {Standard }}$ and $\beta_{\text {Sim. }}$. In this case the results are conclusive since the $\beta_{\text {Standard }}$ exhibit the same performance than $\beta_{\text {Sim }}$ and therefore no bias or significant deviations are observed. These results are consistent with Guijarro et al. (2012) and lead to affirm that both approaches cannot be considered as equivalents since $\beta_{\text {Trad }}$ is biased. 
(a)

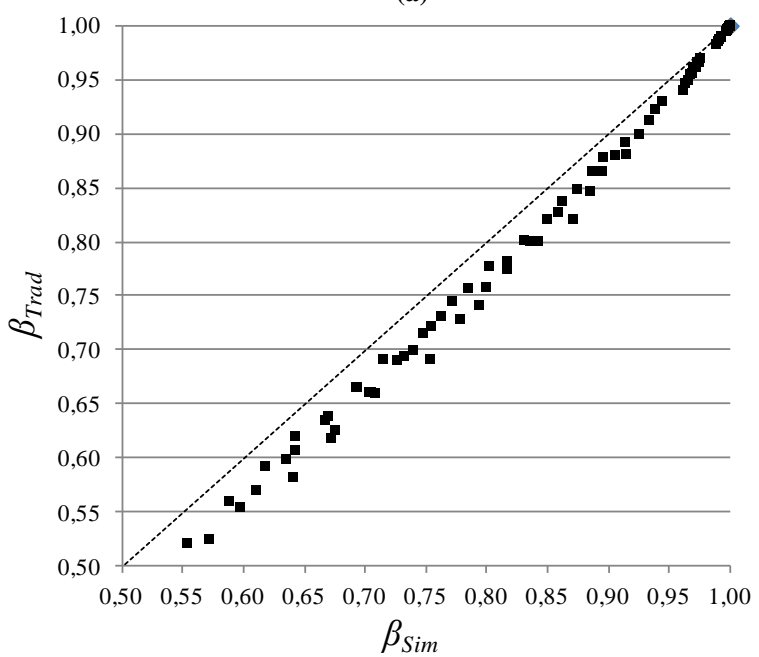

(b)

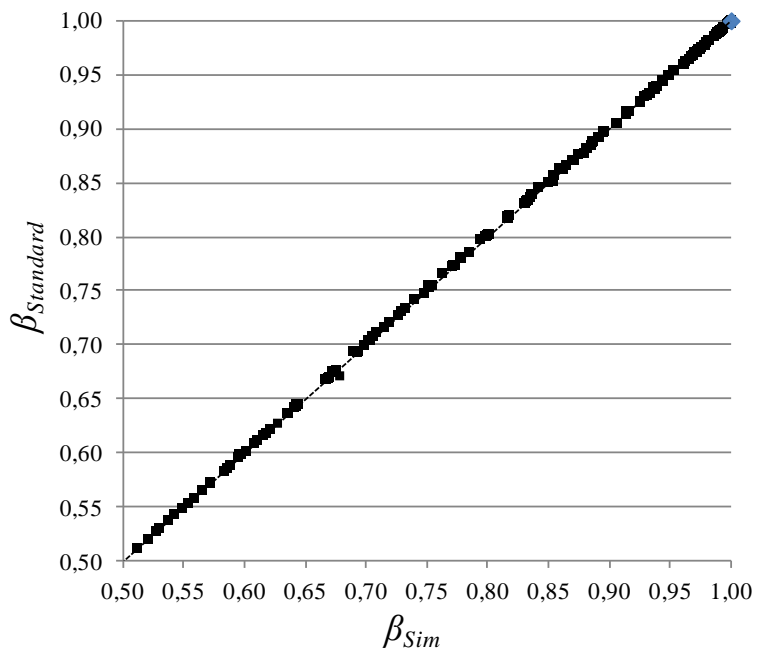

Fig. $2 \beta_{\text {Trad }}$ and $\beta_{\text {Standard }}$ vs. $\beta_{\text {Sim }}$ for the cases from Table 1 .

From a practical point of view, implications which arise from the biased performance of the Traditional approach are found when using a target fill rate to define the control parameters of the inventory policy. Once the order quantity $Q$ is defined, following for example a cost criterion, the reorder point, $s$, can be defined following a service criterion as the fill rate. Then, the question is what happens if managers use a biased method for that purpose. Figure 3 can be used to answer this question. It shows the evolution of the traditional and the standard approaches when increasing the reorder point for a Poisson distributed demand with demand rate $\lambda=2$, order quantity $Q=6$ and lead time $L=3$. If, for example, a target fill rate is set to 0.75 , the traditional approximation leads to $s=5$ whereas in fact just $s=4$ is necessary to reach the target, as shown the standard approach. This example illustrates that using the traditional approximation to determine the reorder point leads to unnecessarily increasing the mean inventory of the system and therefore the holding costs associated to it. This inefficiency is especially relevant in those industries in which the unit cost of the product is very high, the storage space is limited or when dealing with perishables. Therefore, managers should be aware of the risk of using the traditional approximation to set the control parameters of the policy to avoid unnecessary increases in holding costs. 


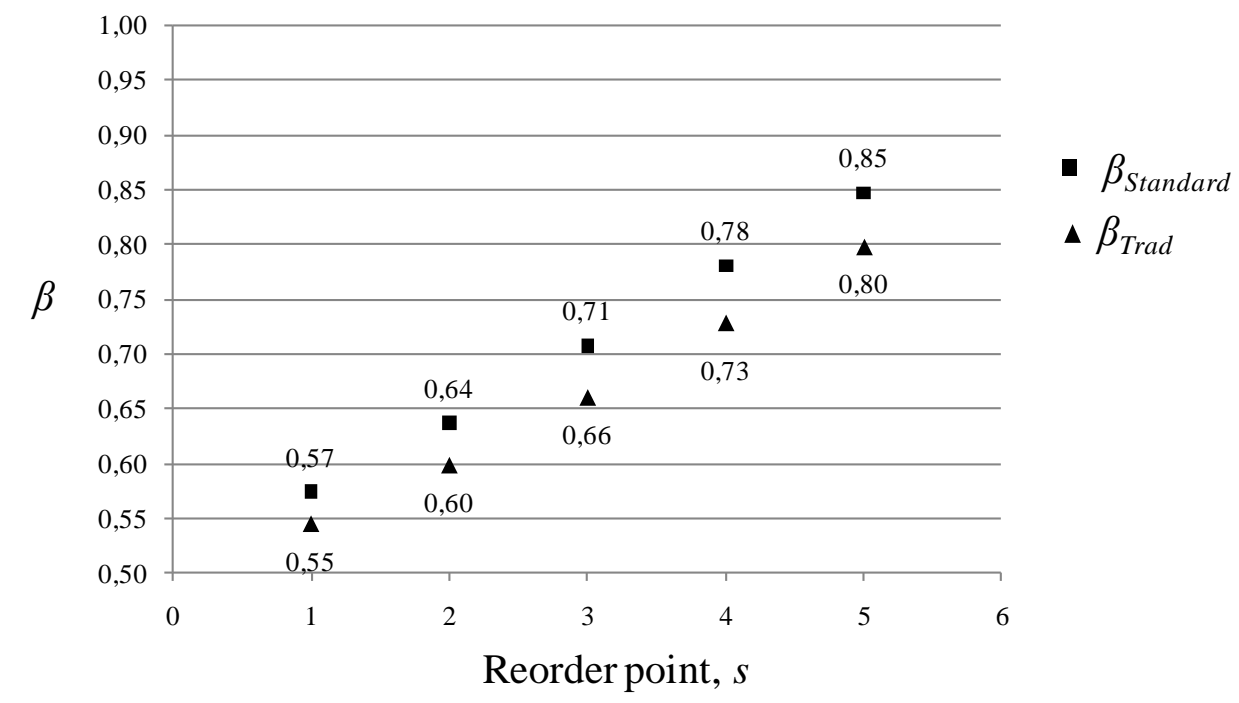

Fig. 3 Comparison between $\beta_{\text {Trad }}$ and $\beta_{\text {Standard }}$ with Poisson demand $(\lambda=2, Q=6, L=3)$.

\section{Summary}

This paper proposes two expressions to calculate the fill rate when the inventory is managed by the continuous review reorder point, order quantity $(s, Q)$ policy in a context of lost sales and discrete demand. Both expressions arise from different interpretations of the fill rate definition. The first approach consists of computing it as the complement of the ratio between the expected unfulfilled demand per replenishment cycle and the expected total demand per replenishment cycle. Following this approach we derive $\beta_{\text {Trad }}$ in section 4.1. The second approach consists of computing the fill rate as the complement of the expected unfulfilled demand over the total demand during the replenishment cycle. Following this approach we derive $\beta_{\text {Standard }}$ in Section 4.2.

Section 5 illustrates the performance of $\beta_{\text {Trad }}$ and $\beta_{\text {Standard }}$ which allows supporting some important results. Regarding $\beta_{\text {Trad, }}$ the results show that it is biased since systematically underestimates the simulated fill rate. Note that an important consequence of the underestimation behaviour is found when $\beta_{\text {Trad }}$ is used to determine the parameters of the policy. If the system is designed to satisfy a target fill rate, the reorder point that will be provided by $\beta_{\text {Trad }}$ will be higher than necessary and therefore it will entail an increase of the mean inventory and consequently an increase of holding costs of the system. Regarding $\beta_{\text {Standard }}$, the results show that it computes accurately the fill rate and therefore it is the appropriate expression in this research context. 


\section{Acknowledgment}

This work was supported by the European Regional Development Fund and Spanish Government (MINECO/FEDER, UE) under the project with reference DPI2015-64133-R.

\section{References}

Agrawal V, Seshadri S (2000) Distribution free bounds for service constrained (Q, r) inventory systems. Nav Res Log 47: 635-656.

Axsäter S (2000) Inventory Control. Kluwer Academic Publishers, Norwell, Massachusetts.

Axsäter S (2006) A simple procedure for determining order quantities under a fill rate constraint and normally distributed lead-time demand. Eur J Oper Res 174: 480-491.

Bijvank M, Vis IFA (2011) Lost-sales inventory theory: A review. Eur J Oper Res 215: 1-13.

Bijvank M, Vis IFA (2012) Lost-sales inventory systems with a service level criterion. Eur J Oper Res 220: 610-618.

Breugelmans E, Campo K, Gijsbrechts E (2006) Opportunities for active stock-out management in online stores: The impact of the stock-out policy on online stock-out reactions". J Retailing 82: 215228.

Diels JL, Wiebach N (2011) Customer reactions in Out-of-Stock situations: Do promotion-induced phantom positions alleviate the similarity substitution hypothsis? Berlin: SFB 649 Discussion paper 2011-021.

Grinstead CM, Snell JL (1997). Introduction to probability. American Mathematical Society.

Guijarro E, Cardós M, Babiloni E (2012) On the exact calculation of the fill rate in a periodic review inventory policy under discrete demand patterns. Eur J Oper Res 218: 442-447.

Gruen TW, Corsten D, Bharadwaj S (2002) Retail Out-of-Stocks: A Worldwide Examination of Extent Causes, Rates and Consumer Responses. Washington, D.C.: Grocery Manufacturers of America. Platt DE, Robinson LW, Freund, RB (1997) Tractable (Q, R) heuristic models for constrained service levels. Manage Sci 43: 951-965. 
Silver EA (1970) A modified formula for calculating customer service under continuous inventory review. AIIE T 2: 241-245.

Silver EA, Pyke DF, Peterson R (1998) Inventory Management and Production Planning and Scheduling. John Wiley \& Sons, Inc.

Tempelmeier H (2007) On the stochastic uncapacitated dynamic single-item lotsizing problem with service level constraints. Eur J Oper Res 181: 184-194.

Vincent P (1983) Practical methods for accurate fill rates. INFOR 21: 109-120.

Zipkin P (2008a) Old and New Methods for Lost-Sales Inventory Systems”. Oper Res 56: 1256-1263.

Zipkin P (2008b). On the structure of lost-sales inventory models”. Oper Res 56: 937-944. 